\title{
Configurações
}

Revista de sociologia

14 | 2014

Para além da Governação. Políticas, práticas e discursos de inclusão e promoção da diversidade cultural

\section{La diversidad lingüística en los espacios virtuales en México: Un análisis crítico desde la Sociedad de la Información}

A diversidade linguística nos espaços virtuais no México: Uma análise crítica a partir da Sociedade da Informação

Linguistic diversity in virtual spaces in Mexico: A critical analysis from the Information Society

Gabriel Pérez Salazar

\section{(2) OpenEdition}

Edición electrónica

URL: http://journals.openedition.org/configuracoes/2278

DOI: 10.4000/configuracoes.2278

ISSN: 2182-7419

Editor

Centro de Investigação em Ciências Sociais

Edición impresa

Paginación: 83-101

ISBN: 1646-5075

ISSN: 1646-5075

\section{Referencia electrónica}

Gabriel Pérez Salazar, « La diversidad lingüística en los espacios virtuales en México: Un análisis

crítico desde la Sociedad de la Información », Configurações [En línea], 14 | 2014, Puesto en línea el 27 marzo 2015, consultado el 01 mayo 2019. URL : http://journals.openedition.org/configuracoes/2278 ; DOI : 10.4000/configuracoes. 2278

Este documento fue generado automáticamente el 1 mayo 2019. 


\title{
La diversidad lingüística en los espacios virtuales en México: Un análisis crítico desde la Sociedad de la Información
}

\author{
A diversidade linguística nos espaços virtuais no México: Uma análise crítica a \\ partir da Sociedade da Informação \\ Linguistic diversity in virtual spaces in Mexico: A critical analysis from the \\ Information Society
}

Gabriel Pérez Salazar

\section{Introducción}

1 La Sociedad de la Información (SI) es un constructo que ha sido abordado desde muy diversas perspectivas. Aunque más adelante desarrollaremos de manera más amplia este concepto, de momento podemos adelantar que se trata, en términos muy generales, de un discurso que otorga a las tecnologías de la información y la comunicación (TIC), el poder para convertirse en el factor decisivo en el desarrollo de áreas como la economía, la política y la educación, entre muchos otros.

2 De manera coincidente con el impulso de la Sociedad de la Información a través de iniciativas promovidas por organismos internacionales como el Banco Mundial, la Organización para la Cooperación y el Desarrollo Económico (OCDE) y la Organización de las Naciones Unidas (ONU); durante la primera década del siglo XXI, se presentaron numerosos análisis críticos que señalaron algunas de las falacias ocultas en este planteamiento. Trabajos como los de Mattelart (2002), Miége (2002) y Crovi (2002) destacaron las estructuras económico-políticas subyacentes a las propuestas emprendidas por una gran cantidad de gobiernos (particularmente de países en vías de desarrollo), relativas al llamado tránsito hacia la SI. Estos autores, identificaron la existencia de una 
serie de problemas y limitaciones relacionadas con las políticas públicas emprendidas con la intención de implantar este modelo: desigualdad en el acceso a Internet, condiciones contextuales inequitativas y de manera particular, grandes deficiencias en el desarrollo de lo que actualmente un sector de académicos identifica como competencias tecnológicas (Cabello y Moyano, 2006; Raposo, Fuentes y González, 2006).

Con este contexto como punto de partida, en el presente trabajo haremos una revisión de algunas de las principales políticas públicas emprendidas desde la visión de la Sociedad de la Información del gobierno federal mexicano, con la diversidad como eje analítico general, pero centrado de forma particular en la diversidad lingüística. Con base en una breve discusión sobre dicha categoría conceptual, se describirán las estrategias emprendidas desde el Sistema Nacional e-México para la inclusión a la SI. Partimos de la hipótesis general (H1) de que la diversidad y la Sociedad de la Información han sido asumidas de tal manera por el gobierno mexicano, que se han dejado de atender aspectos muy relevantes en torno a variables como las ya mencionadas competencias tecnológicas, la creación de contenidos y la diversidad étnico-cultural presente en México; y que por lo tanto, ha sido de la sociedad civil, y no de las agencias gubernamentales de donde parten las iniciativas que dan presencia a las lenguas indígenas en el ciberespacio.

\section{Diversidad en México}

4 La diversidad es una categoría analítica en las Ciencias Sociales, que como Ritzer (2007) sugiere, se refiere a la forma en que las unidades de análisis se distribuyen al interior de una población determinada, dando lugar a una variedad que oscila entre lo amplio y lo reducido, en los valores que es posible observar. Así, es posible hablar de una gran cantidad de aspectos que pueden ser considerados para abordarla, entre las que es posible mencionar: etnia, religión, lengua, edad y otros descriptores similares. Sin embargo, como Geertz (1996) sugiere, muchos planteamientos elaborados en torno a esta categoría, frecuentemente son englobados bajo la etiqueta de diversidad cultural. Es claro que estas variedades se ubican en un plano complejo, en el que se entrecruzan aspectos que pertenecen tanto a lo grupal como a lo individual, en una relación dialógica entre el somos y lo que son los otros (Todorov, 2007), a partir de sentidos de pertenencia y valoraciones subjetivas que median en toda interacción social.

5 La problematización de la diversidad, en términos de acciones concretas, es presentada por Geertz (1996) como la ubicación del sujeto en relación con aquello que le es ajeno, y que lleva implícita una capacidad de reconocimiento del otro desde su propia realidad. En otras palabras, podemos decir que se trata de relaciones intersubjetivas (Fernández Chriestib, 2013) en las que las variaciones de lo distinto son el eje de articulación. Así, se tienen varios planos posibles para el abordaje de la diversidad, que van desde aspectos muy sutiles de variación individual, hasta lo que es distinto en relación con lo grupal.

De esta forma, plantear el asunto de la diversidad en cualquier contexto, metodológicamente hablando, implica inevitablemente una selección de variables, que por la ya mencionada naturaleza compleja del fenómeno, serán siempre insuficientes para abordar la amplitud de la realidad social, así sea en un espacio concreto. Por su configuración histórica, en el caso de México, dicha delimitación suele circunscribirse a aspectos relacionados con la diversidad étnica, frecuentemente asociados a sus expresiones lingüísticas (Hernández, 1983; Bartolomé, 1997); aunque más recientemente se han agregado otras variables como la identidad sexual (Szasz, 1998) y la religión (de la 
Torre y Gutiérrez, 2007). Con base en Díaz-Couder (1998), en el presente trabajo tomaremos la lengua ${ }^{1}$ como un indicador de dicha diversidad cultural, debido a su naturaleza estructurante (Bourdieu, 2006); es decir, como una mediación entre el sujeto y las relaciones que establece al interior del campo en el que se ubica.

7 Desde esta perspectiva, INEGI (2010a) reporta un total de 92 lenguas indígenas que son habladas por prácticamente 6,7 millones de personas en México ${ }^{2}$. En la Tabla 1 se muestran las diez con mayor número de hablantes, entre las que su ubica el náhuatl en el primer lugar.

Tabla 1.

\section{LENgUAS INDígENAS EN MÉXICO Y HABLANTES (DE 5 AÑoS Y MÁS)}

\begin{tabular}{|l|r|r|}
\hline Lengua & Hablantes & Porcentaje \\
\hline Náhuatl & 1544968 & $23,08 \%$ \\
\hline Maya & 786113 & $11,74 \%$ \\
\hline Mixteco & 471710 & $7,05 \%$ \\
\hline Tzeltal (Tseltal) & 445856 & $6,66 \%$ \\
\hline Zapoteco & 425123 & $6,35 \%$ \\
\hline Tzotzil (Tsotsil) & 404704 & $6,04 \%$ \\
\hline Otomí & 284992 & $4,26 \%$ \\
\hline Totonaca (Totonaco) & 244033 & $3,64 \%$ \\
\hline Mazateco & 223073 & $3,33 \%$ \\
\hline Chol (Ch'ol) & 212117 & $3,17 \%$ \\
\hline Otras & 1652539 & $24,68 \%$ \\
\hline Total & 6695228 & $100,00 \%$ \\
\hline
\end{tabular}

Fuente: INEGI (2010a).

Por entidad federativa (Tabla 2), es claro que este es un factor de diversidad cultural que resulta particularmente relevante en la región sureste de México.

TABLA 2.

SELECCIÓN DE ENTIDADES FEDERATIVAS CON MAYOR CANTIDAD DE HABLANTES

DE LENgUAS INDÍgENAS (5 AÑoS Y MÁs) 


\begin{tabular}{|c|c|c|c|c|}
\hline Entidad federativa & $\begin{array}{l}\text { Población } \\
\text { que habla } \\
\text { lengua indi- } \\
\text { gena }\end{array}$ & Porcentaje & $\begin{array}{l}\text { Población } \\
\text { que no ha- } \\
\text { bla } \\
\text { español }\end{array}$ & Porcentaje \\
\hline Oaxaca & 1165186 & $34,20 \%$ & 188230 & $16,20 \%$ \\
\hline Yucatán & 537516 & $30,30 \%$ & 40273 & $7,50 \%$ \\
\hline Chiapas & 1141499 & $27,20 \%$ & 371315 & $32,50 \%$ \\
\hline Quintana Roo & 196060 & $16,70 \%$ & 8867 & $4,50 \%$ \\
\hline Guerrero & 456774 & $15,10 \%$ & 134797 & $29,50 \%$ \\
\hline Hidalgo & 359972 & $15,10 \%$ & 43991 & $12,20 \%$ \\
\hline A nivel nacional & 6695228 & $6,70 \%$ & 980894 & $14,70 \%$ \\
\hline
\end{tabular}

FUENTE: ELABORACIÓN A PARTIR DE INEGI (2010B)

Ante este panorama, resulta pertinente destacar que aunque a nivel nacional el porcentaje de personas que hablan una lengua indígena es de 6,7\%, en Estados como Oaxaca (donde hay una importante presencia de hablantes de zapoteco) y Yucatán (lengua maya), la prevalencia es de alrededor de la tercera parte de su población (34,2 y $30,3 \%$, respectivamente). Con base en estas cifras, es notable que muchos de ellos hablan además el español (la mitad en Oaxaca y casi tres cuartas partes en Yucatán); situación que contrasta marcadamente con la situación que prevalece en Chiapas y Guerrero, donde casi la tercera parte «de quienes hablan una lengua indígena, carecen del dominio del español. Esto recalca la importancia que tiene esta variable (la lengua), dentro del mosaico de la diversidad cultural en México.

\section{La Sociedad de la Información como discurso tecnodeterminista y panorama general del Sistema Nacional e-México}

10 El propósito de este apartado es hacer una revisión crítica de la Sociedad de la Información, como contexto general a las políticas públicas emprendidas en materia de diversidad lingüística en línea. La estrategia expositiva consiste en iniciar con la presentación de sus antecedentes, para luego dar paso a una discusión que permita profundizar en sus principales características, haciendo énfasis en la manera en que éstas han derivado en el desarrollo de acciones técnico-instrumentales, que no han tomado en cuenta las dimensiones socio-culturales de los procesos de equipamiento tecnológico en México.

\subsection{El Consenso de Washington}

11 Autores como Crovi (2002) y Bergonzelli y Colombo (2006) ubican este evento como el hito que dio lugar al posterior establecimiento de la Sociedad de la Información como modelo, basado en las TIC como factor determinante en el progreso y desarrollo. Se trató de una ronda de trabajos a la que acudieron durante 1990, "un grupo de personas responsables 
de las políticas de la región de América Latina y el Caribe, representantes de organismos internacionales y miembros de comunidades académicas y centros de estudios" (Burki y Perry, 1998: 1). La intención era analizar las reiteradas recesiones en las que se habían visto envueltas muchas de las economías emergentes en África, Asia y América Latina entre las décadas de 1970 y 1980, con el fin de establecer estrategias que lograran revertirlas.

Fue a partir de estos trabajos cuando, además de impulsar un conjunto de acciones de intervención económica ${ }^{3}$, se delinearon políticas generales en cuanto a la adopción de las tecnologías de la información y la comunicación, como base para el desarrollo de dichas regiones (Crovi, 2002). Como veremos enseguida, esta concepción de la tecnología como motor del desarrollo, tiene un conjunto de antecedentes académicos muy claros en las ciencias económicas, que consideramos oportuno revisar en función de su impacto en la forma en que fue desarrollada la visión hegemónica en torno a la Sociedad de la Información.

Desde la década de 1960, se establecieron diversas hipótesis en torno al progreso logrado por diversos países (como Japón y Corea del Sur) luego de la Segunda Guerra Mundial, que en relativamente poco tiempo habían alcanzado altos niveles de desarrollo. Uno de estos planteamientos teóricos recibió el nombre de brecha tecnológica, y ha sido descrita desde la Economía de la siguiente manera:

El enfoque de la brecha tecnológica desarrollado por Posner (1961), Gomulka (1971), Cornwall (1977), Abramovitz (1986) y otros autores plantea que los significativos diferenciales en los niveles y tendencias tecnológicos que caracterizan al sistema económico internacional sólo podrán ser remontados mediante transformaciones radicales en las estructuras tecnológicas, económicas y sociales. El enfoque supone que existe una estrecha correlación entre las tasas de crecimiento económico y las tasas de crecimiento del nivel tecnológico. El relativo atraso tecnológico de los países pobres conlleva una oportunidad para su rápido crecimiento económico. (Guzmán y Gómez, 2010: 41)

Con este antecedente conceptual, las directivas establecidas por el Banco Mundial, el Fondo Monetario Internacional y otros organismos internacionales que ya han sido mencionados; dieron lugar a la consolidación de una relación simbólica entre desarrollo y tecnología, que según Mattelart (2002), había iniciado desde el siglo XVIII durante la llamada Era de la Razón. Es en este periodo cuando se plantea en términos generales la idea de que el progreso de la humanidad responde a un modelo lineal, en el que "los avances de la ciencia y la mecánica se traducen en el mejoramiento general de la condición humana" (Marx, 2001: 139), y que se encuentra presente de manera transversal en el planteamiento hecho en torno a la Sociedad de la Información como ha sido aplicada en México.

\subsection{La Sociedad de la Información}

Aunado a las propuestas económicas derivadas del Consenso de Washington, la Sociedad de la Información cuenta con una serie de bases conceptuales que desde la década de 1960, habían abordado los cambios que ha significado el pasar de un modelo productivo de carácter fundamentalmente industrial en los países desarrollados, a una economía en la que la mayor parte de la riqueza es generada por el sector de los servicios (financieros, informáticos, etc.). 

Postindustrial de Mumford (1967) y Bell (1976); la Era del Conocimiento, introducida por Fritz Machlup en 1962 y retomada por Peter Drucker en 1993 (en Gómez Palacio, 1998); así como en la Sociedad Superindustrial de Toffl er (1993). En todos ellos, se enfatiza la importancia que tiene la tecnología como base del desarrollo, y se da lugar a propuestas como las de Negroponte (1995), quien es uno de los defensores más visibles de la visión optimista en torno a la Sociedad de la Información, al afirmar que la digitalización tiene una naturaleza motivadora, que hará que cada vez más personas en todo el mundo se sumen a la superautopista de la información para aprovechar sus beneficios.

A partir de todo ello, podemos decir que si bien la Sociedad de la Información como marco conceptual es planteada como tal hasta inicios de la década de 1990, cuenta con una serie de antecedentes que permiten entender su sentido general en torno al uso de las tecnologías, así como identificar el aparato ideológico neoliberal del cual surge. El pensamiento economicista, por lo tanto, ha jugado un papel muy importante en la forma en que esta visión se convirtió en una estructura de acción impuesta a los países en vías de desarrollo como México. Esto coincide con la descripción que Becerra y Mastrini hacen de la Sociedad de la Información, en la que la relacionan con el ya mencionado modelo derivado del Consenso de Washington:

La sociedad de la información se halla articulada por el dominio de tres ideas fuerza: la desregulación, la liberalización y la integración competitiva del planeta como escenario de realización de la economía de mercado. En un nive económico, el proyecto argumenta que la centralidad de la información y la comunicación en la estructuración de las sociedades contemporáneas permite la obtención de mayores beneficios y un salto en la productividad, con el consecuente crecimiento de las economías. En el plano de lo social, los organismos y gobiernos mencionados enfatizan beneficios como consecuencia de la diseminación y ubicuidad de las tecnologías convergentes de información y comunicación. (Becerra y Mastrini, 2004: 98)

sde una perspectiva crítica podemos decir que el modelo de desarrollo que implica la Sociedad de la Información, ha sido aplicado de manera que se ha concentrado sólo en lo tecnológico y ha dejado a lo económico y social como variables dependientes. Como ya ha sido señalado, las políticas públicas basadas en esta visión se han centrado en la idea de que un incremento en el acceso a las TIC, prácticamente por sí mismo, pondría a la disposición de los ciudadanos grandes cantidades de información que impactarían de manera positiva en áreas como la educación, la democracia, los sistemas de salud, y, sobre todo, en la economía. Como Warschauer señala, esta es la esencia del tecnodeterminismo; es decir "la creencia en que la mera presencia de la técnica conduce a sus aplicaciones ordinarias, las cuales a su vez, traen consigo el cambio social" (2003: 20). Así, en México se desarrolla el Sistema Nacional e-México, a partir de $2000^{4}$, con la intención de promover el acceso a la Sociedad de la Información.

19 Esta iniciativa gubernamental fue estructurada con base en tres ejes principales: 1) la construcción de una infraestructura nacional de telecomunicaciones de alta velocidad, 2) el establecimiento de una red nacional de centros públicos de acceso, conocidos como Centros Comunitarios Digitales (CCD), y 3 ) la articulación de todos estos esfuerzos a través de un portal web, donde además se enlazaran contenidos para los ciudadanos, catalogados en una serie de subportales temáticos que describiremos más adelante.

Luego de diversos estudios (Pérez Salazar, 2004 y 2007; Pérez Salazar y Carabaza, 2011), hemos llegado a la conclusión de que los resultados alcanzados entre 2000 y $2012^{5}$ para 
dicha iniciativa gubernamental, quedaron muy lejos de las metas establecidas. La primera línea de acción jamás fue puesta en marcha, y en la actualidad México sigue careciendo de una infraestructura propia de alta velocidad ${ }^{6}$ en materia de conectividad a Internet. En cuanto a los CCD, si bien se logró la instalación de alrededor de 8 mil de estos lugares de acceso a nivel nacional; muchos de ellos fueron establecidos en puntos de acceso no públicos, como centros de cómputo en escuelas de educación básica, y al interior de hospitales del Sector Salud. Una buena parte de los restantes, fueron colocados en bibliotecas públicas, que no contaban con una estrategia de soporte económico que posibilitara su funcionamiento en el largo plazo, por lo que al cabo de unos meses, más del $50 \%$ de ellos carecían de acceso a Internet.

21 Además, en estos sitios no se habían desarrollado planes para la sustitución de los equipos que fueron siendo dañados, tanto por las condiciones normales de uso, como por su eventual obsolescencia tecnológica (Santos, de Gortari, Angulo, Rueda y Pérez Salazar, 2006). Las acciones encaminadas al desarrollo de competencias digitales, salvo casos aislados emprendidos por iniciativas individuales, fueron nulas como acción concertada.

Otro asunto que es pertinente resaltar en la manera en que fue ejecutado el Sistema Nacional e-México, tiene que ver con el tipo de software que sería utilizado en los equipos de cómputo con que serían equipados los CCD. En lugar de optar por sistemas de software libre $e^{7}$, la Secretaría de Comunicaciones y Transportes, responsable de este proyecto, hizo un convenio con la empresa estadounidense Microsoft para este fin. Las restricciones inherentes al software propietario de dicha empresa, tienen un impacto directo en el reconocimiento a la diversidad cultural en México, en virtud de que, a diferencia de lo que hubiera sido posible en sistemas de software libre (Jiménez y Gutiérrez, 2010), jamás se desarrollaron plataformas en lenguas distintas al español.

\section{Diversidad lingüística y el portal del Sistema Nacional e-México}

Esta parte del trabajo de campo está sustentada en un análisis de contenido al portal del Sistema Nacional e-México, elaborado a partir del modelo de Krippendorff (1990). Se hizo un registro de los 2352 enlaces que integraban el contenido de dicho portal, de manera que se integró una matriz que permitía identificar, entre otros indicadores, el tipo de contenido específico al que conducía y el idioma en que aparecía. Este registro fue elaborado en dos ocasiones: en febrero de 2004 y en febrero de 2010; lo que permitió determinar que la estructura presente entre ambas observaciones (correspondientes a dos administraciones del gobierno federal distintas), era fundamentalmente la misma.

El portal web del Sistema Nacional e-México, durante el periodo 2000- 2012, estuvo organizado a partir de dos grandes secciones específicas: 1) Comunidades y 2) Subportales. Mientras que los subportales tenían un acomodo temático de la información por sectores como educación, salud, economía y gobierno; las llamadas comunidades representan una segmentación de la población, a partir de los siguientes grupos: a) Adultos en plenitud ${ }^{8}, \mathrm{~b}$ ) Campesinos, c) Empresarios, d) Estudiantes, e) Indígenas, f) Migrantes, g) Mujeres, h) Niños, i) Visitantes.

Como es posible observar, hay diversas variables involucradas en la conformación de dichas comunidades. Mientras que Adultos en plenitud y Niños atienden a dimensiones relacionadas con grupos de edades, Campesinos, Empresarios y Estudiantes, son variables 
ocupacionales. Visitantes se refiere a información turística, y Migrantes se limitaba a dar enlaces relativos a información consular y sugerencias generales en torno al envío de remesas. Mujeres pertenece a una variable relacionada con el género y sólo la comunidad Indígenas, tiene relación con lo que ya hemos planteado en torno al contexto étnico mexicano. Al menos en la estructura formal del portal de e-México, tal es la manera en que se observa un muy somero acercamiento a la diversidad cultural.

Una mirada más cuidadosa al sub-portal Indígenas, nos permite observar que de los 2352 enlaces que integraban el directorio completo de e-México durante el periodo analizado, dicha sección estaba integrada por 47 vínculos, es decir, el 2,0\% en relación con este total. De estos 47 enlaces, sólo uno conducía a contenidos disponibles en lengua indígena ${ }^{9}$, lo que representa el 0,04\% del total de enlaces del portal de e-México. Las secciones que integraban el sub-portal se muestran en la Tabla 3, ninguna de las cuales hacía referencia a las lenguas indígenas.

Tabla 3.

SECCIONES DEL SUB-PORTAL INDÍgENAS EN E-MÉXICO

\begin{tabular}{|l|c|}
\hline & $\begin{array}{c}\text { Cantidad } \\
\text { de enlaces }\end{array}$ \\
\hline Arte y Cultura & 13 \\
\hline Comercialización de Productos & 4 \\
\hline Derechos Humanos & 4 \\
\hline Educación y Capacitación & 11 \\
\hline Eventos y Festivales & 2 \\
\hline Grupos étnicos & 2 \\
\hline Instituciones y Programas de Apoyo & 9 \\
\hline Vivienda, Servicios y Albergues & 2 \\
\hline Total & 47 \\
\hline
\end{tabular}

\section{FUENTE: ELABORACIÓN PROPIA}

Reiteramos que, además del ya mencionado, ningún otro enlace del portal de e-México conducía a contenido alguno disponible en lengua indígena. Es evidente que, al menos desde esta iniciativa, era prácticamente nulo el respeto a la diversidad lingüística, a pesar de ser un derecho consagrado en la Ley General de Derechos Lingüísticos para los Pueblos Indígenas (LGDLPI), promulgada en marzo de 2003, durante la misma administración federal responsable del lanzamiento del Sistema Nacional e-México. De forma específica, la selección de contenidos del portal de e-México, contraviene lo señalado en los párrafos II y III del Art. 13 de dicha Ley:

ARTícUlO 13. Corresponde al Estado en sus distintos órdenes de gobierno la creación de instituciones y la realización de actividades en sus respectivos ámbitos 
de competencia, para lograr los objetivos generales de la presente Ley, y en particular las siguientes:

[...]

II. Difundir en las lenguas indígenas nacionales de los benefi ciarios, el contenido de los programas, obras y servicios dirigidos a las comunidades indígenas;

III. Difundir a través de los medios de comunicación las lenguas indígenas

nacionales de la región para promover su uso y desarrollo (LGDLPI, 2003).

Es de esta manera, que las iniciativas gubernamentales del orden federal han dejado de lado en sus contenidos y estrategias en línea, la diversidad lingüística implícita en estos 92 idiomas hablados en México.

\section{La Sociedad de la Información desde la sociedad civil}

Con base en los datos antes mostrados, es claro que la Sociedad de la Información, al menos como política pública, no se ha traducido en acciones de las entidades gubernamentales que sean suficientemente congruentes ni con la legislación mexicana en materia de derechos lingüísticos, ni con el respeto a la diversidad cultural del país. La administración federal 2012-2018 encabezada por Enrique Peña Nieto, y su Estrategia Digital Nacional ${ }^{10}$, al menos a partir de la información que mostraba al momento de elaborar el presente trabajo, omite en sus documentos, estrategias específicas que contemplen el desarrollo de contenidos en lenguas indígenas disponibles en línea. A nivel estatal, la situación no es diferente. Con base en una observación de los elementos presentes en los portales de los gobiernos estatales que tuvieran la mayor proporción de hablantes de lengua indígena mostradas en la Tabla 2, durante febrero de 2014, se verificaron los idiomas en que eran mostrados sus contenidos. De esta forma, se constató que los sitios web de los estados de Oaxaca, Yucatán, Chiapas, Quintana Roo ${ }^{11}$, Guerrero e Hidalgo, presentan información para sus ciudadanos exclusivamente en español. Ninguno de estos sitios ofrece la posibilidad de consultar sus contenidos en las lenguas presentes en sus regiones, ni siquiera las que tienen mayor cantidad de hablantes, como el náhuatl, el maya o el zapoteco.

Este panorama caracterizado por la inacción gubernamental, ha coincidido con acciones concretas llevadas a cabo por algunos sectores de la sociedad civil. Así, es posible encontrar en la Wikipedia, una versión en náhuat $1^{12}$, curada por colaboradores sin ninguna adscripción ni apoyo gubernamental. Con el fin de profundizar en las motivaciones de quienes colaboran en esta versión de la Wikipedia, se aplicó una entrevista en profundidad a cinco de ellos. La muestra fue seleccionada a partir de los usuarios con mayor cantidad de artículos recientemente escritos en la Huiquipedia (Wikipedia en náhuatl), quienes fueron contactados de manera directa por el autor de este trabajo, a través de sus perfiles públicos en dicha plataforma. Las entrevistas fueron realizadas a través del correo electrónico durante el mes de agosto de 2014.

31 A partir de dichas entrevistas, podemos destacar que las motivaciones que expresaron para colaborar, tienen que ver principalmente con la preservación y difusión de esta lengua, que consideran como una parte importante de la cultura mexicana; a pesar de que sólo dos de ellos tienen al náhuatl como su lengua materna. Para enfatizar la naturaleza no gubernamental de este trabajo, a pregunta expresa, los cinco entrevistados 
coincidieron en no haber recibido nunca ningún apoyo económico, de ninguna entidad de gobierno en relación con esta actividad.

En lo que respecta al software libre, destacan las acciones emprendidas por el grupo Mozilla México ${ }^{13}$, que ha desarrollado y puesto a la disposición de los usuarios de forma gratuita, el navegador Firefox en 26 lenguas indígenas mexicanas ${ }^{14}$. Entre algunas de ellas es posible mencionar el náhuatl (en dos variantes), el mixteco (cuatro variantes), el mazateco (dos variantes), maya, purépecha, rarámuri y yaqui.

Si bien la creación de contenidos y aplicaciones son indicadores de su relevancia social, el empleo activo de lenguas autóctonas en el ciberespacio, es una evidente forma de preservación por parte de tales sectores se la sociedad civil, que son quienes finalmente poseen y ejercen este bagaje cultural. Tal es el caso de su uso en las herramientas para la administración de redes sociales en línea (en adelante, simplemente redes sociales), entre las que por su visibilidad y relevancia destaca Facebook ${ }^{15}$. A partir de una búsqueda de grupos en esta plataforma bajo la técnica de la bola de nieve ${ }^{16}$, fue posible localizar los grupos Hablemos Náhuat ${ }^{17}$, Queremos hablar náhuat ${ }^{18}$, y Yo soy mexica ${ }^{19}$; en los que se promueve y practica esta lengua. Luego de un seguimiento exploratorio a estos foros, fue posible observar que una de las intenciones presentes en sus miembros, es la divulgación de algunas de las reglas gramaticales y léxico básicos del náhuatl, empleando de manera muy frecuente memes ${ }^{20}$, como los que se muestran en la Figura 1.

Grupos similares fueron localizados en esta misma red social ${ }^{21}$, en torno a otras de las lenguas mencionadas en la Tabla 1, de las que mostramos una selección de aquellas con mayor número de seguidores y/o mayor actividad del grupo:

Figura 1.

Memes de divulgación del náhuatl

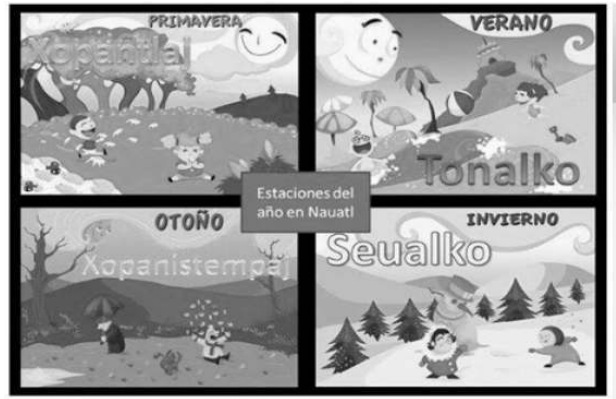

Frases de cortesía

Buenos dias = ijnalti, tonalti, pialli, kualli tonalti.

Buenas tardes $=$ tiotlakilti, tiotlakti, kualli tiotlakti.

Buenas noches $=$ youalti, tlayoualti, kualli yoaualti.

Con permiso $=$ nimitsixpanos. (te voy a pasar en tu frente)

Pase usted $=$ ximopanoti.

¿Cómo estás? $=$ ¿kenijkatsa tiitstok?

¿Cómo te llamas? = katsa motokax?, ¿tlen motokax?

¿De dónde vienes? = ¿kampa tiuala?

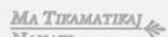

$\Rightarrow \frac{\text { MA TIRAMATDEN }}{\text { NAUATL Heblemor Nahioat }}$

FUENTE: CAPTURAS DE PANTALLA EN LOS gRUPOS HABLEMOS NÁHUATL Y QUEREMOS HABLAR NÁHUATL.

- Maya: Lengua Maya ${ }^{22}$, Lengua maya yucateca ${ }^{23}$ y Aprendiendo el lenguaje maya quiche ${ }^{24}$;

- Tzeltal: Snopel batzil kop aprendiendo tzeltal ${ }^{25}$ y San Marcos Tulija ${ }^{26}$;

- Zapoteco: Zapoteco para principiantes ${ }^{27}$ y Zapoteco Petapa ${ }^{28}$;

- Totonaca: Yolkualtakamej A C Bondadoso ${ }^{29}$;

- Mazateco: Xota Ndi'ya (Mazatecos) ${ }^{30}$. 
manera general, en estos espacios fue posible observar memes como los ya mencionados, así como lecciones gramaticales básicas y conversaciones en estas lenguas indígenas entre sus integrantes y seguidores. Sin embargo, en casos como los que se muestran en las Figura 2 y 3, el sentido va más allá de lo meramente idiomático, alcanzando aspectos que reflejan un sentido de relevancia y responsabilidad social mucho más amplio.

Figura 2.

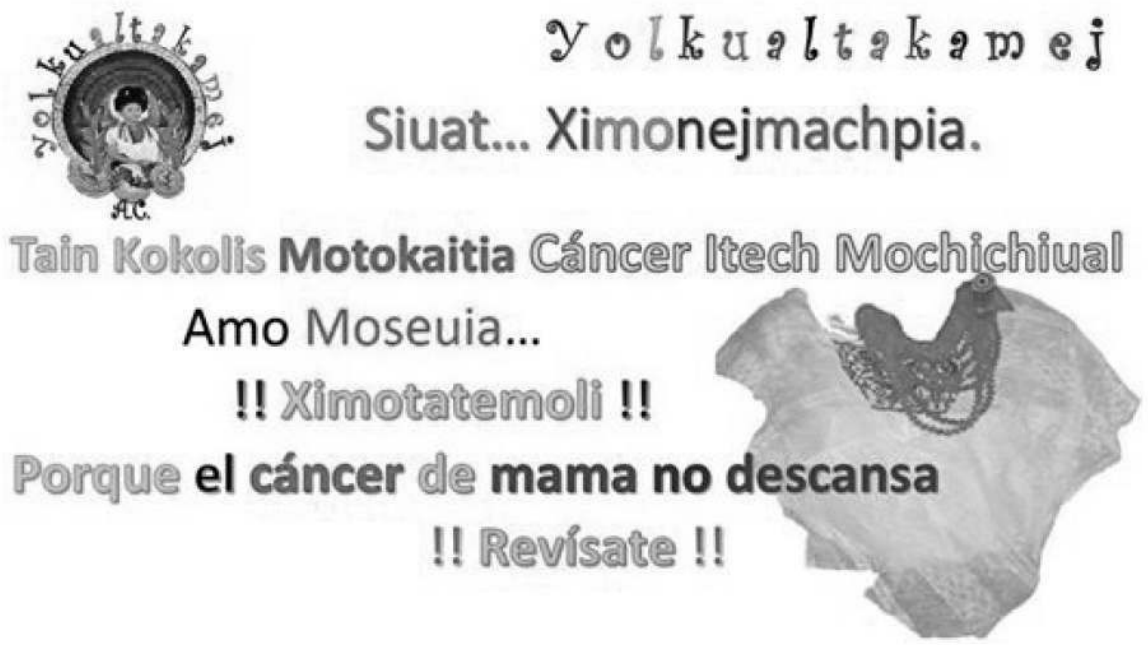

FUENTE: CAPTURA DE PANTALLA de LA PÁgINA OLKUALTAKAMEJ a C BONDAdOSO.

Figura 3.

RESIgNifi CACIÓN DEL HABLANTE DE ZAPOTECO

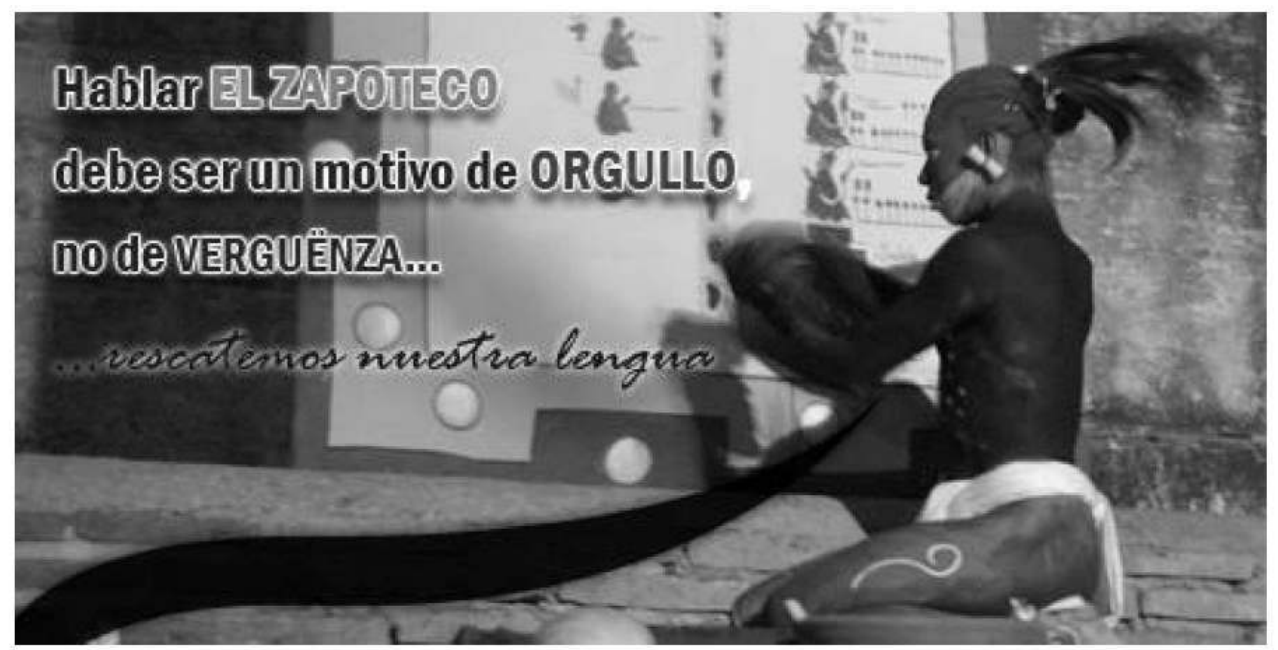

FUENTE: CAPTURA DE PANTALLA DE LA PÁgINA ZAPOTECO PETAPA

\section{Consideraciones finales}

41 La creencia en que la mera presencia de las TIC conduciría al desarrollo social y económico, se relaciona de manera muy importante con la aplicación de políticas públicas parciales y tecnodeterministas. Se trata de visiones que, lejos de considerar el resultado 
de estudios hechos desde muy diversos sectores de la academia y la complejidad misma del fenómeno, pueden ser entendidos a partir de estrategias de corto plazo en el plano de la política, entendida en la más instrumental de sus aplicaciones. Así, durante la primera década del siglo XXI, algunas de las principales acciones llevadas a cabo en México en relación con el tránsito a la Sociedad de la Información, se centraron en simplemente repartir equipos de cómputo en sitios de acceso público, sin considerar de manera suficientemente clara, por qué ni para qué; esto es, más allá de los fácilmente deducibles intereses tejidos alrededor de las industrias de este sector y del lucimiento de los funcionarios en turno.

En referencia a las dimensiones económicas y sociales planteadas por el enfoque dado por la brecha tecnológica que mencionamos en la parte inicial de este trabajo, no pudimos observar estrategias encaminadas a atender estas otras dimensiones que la tecnología implica siempre, en aspectos como la construcción de competencias tecnológicas, o el respeto a la diversidad cultural. En otras palabras, lo que debería haber sido abordado desde un enfoque complejo, que incluyera aspectos socio-culturales y educativos, fue tratado como un problema estrictamente tecnológico, que en la mayor parte de los casos sólo contempló la entrega de equipos de cómputo.

Iniciativas como el Sistema Nacional e-México (2000-2012), y su continuación, la Estrategia Digital Nacional emprendida por Enrique Peña Nieto; no han planteado formas claras y precisas para atender el respeto a la diversidad lingüística en México. A pesar de que hay un relevante sector de la población que sólo habla alguna lengua autóctona, así como de la existencia de la Ley General de Derechos Lingüísticos para los Pueblos Indígenas (2003); los contenidos generados por las instancias gubernamentales que fueron analizadas, se presentan en idioma español de forma casi exclusiva. La única excepción encontrada en la muestra, está dada por el portal del estado de Quintana Roo, que posiblemente a partir de su importancia como destino turístico ${ }^{31}$, ofrece la posibilidad de desplegar los contenidos de dicho sitio web en idiomas extranjeros (inglés, alemán, italiano, francés y ruso); pero no así en función del 16,7\% de su población maya-hablante.

Esta situación, caracterizada por una evidente omisión de dichas entidades de gobierno; ocurre a la par de acciones llevadas a cabo por diversos sectores de la sociedad civil, en torno al ejercicio de sus derechos culturales. Sin apoyo gubernamental alguno, se han construido contenidos y sistemas que son un intento por reducir esta brecha cultural en línea y de alguna manera reconocer y preservar la diversidad lingüística presente en territorio mexicano. Como fue observado en la parte final del trabajo de campo, a esto debe sumarse el uso de las redes sociales, como espacios que son usados activamente para la promoción y conservación de una gran cantidad de lenguas autóctonas. Se trata de espacios de alta significación social, en los que se emplean estos códigos comunicativos estructurantes, como parte de las expresiones culturales de tales grupos, en ámbitos que en ocasiones rebasan lo estrictamente lingüístico, y que atienden a situaciones y problemáticas de la mayor relevancia.

\section{Hacia una agenda de la diversidad lingüística en el ciberespacio}

Los derechos de quienes en México hablan una lengua distinta al español, se encuentran reconocidos en la ya mencionada LGDLPI de 2003. El gobierno mexicano, en sus tres niveles, está por ello obligado a respetarlos. No obstante, como sucede con muchas otras leyes en este país, su aplicación no es ejercida con suficiente claridad. La diversidad 
cultural presente en México, conlleva una serie de derechos que no están siendo respetados de manera precisa, poniendo en peligro esta riqueza. Contrariamente a las preocupaciones que pudieran surgir en algunos sectores bienintencionados, el uso de las TIC, lejos de dañar o contaminar el bagaje cultural de los pueblos autóctonos; está operando como un vehículo de visibilización, promoción y conservación de sus lenguas y tradiciones. Dadas las características operativas de dichas mediaciones, esto además ocurre en situaciones que trascienden las limitaciones espacio-temporales inherentes a los procesos presenciales.

Por todo ello, y con base en los resultados alcanzados en este trabajo exploratorio, consideramos que es necesario el establecimiento de estrategias en materia de políticas públicas, que sean coherentes en la ya citada Ley. Cada portal de gobierno, en tanto instrumento de relación con la administración pública, debe ofrecer la posibilidad de tener acceso a contenidos, trámites y otras formas de relación con el Estado, al menos en las lenguas indígenas con mayor presencia, tanto a nivel nacional, como estatal y municipal; dependiendo de las condiciones particulares de cada región. Más allá de promover el simple acceso a las TIC, el tránsito a una Sociedad de la Información realmente incluyente, tiene que ver con la construcción de contenidos acordes a las condiciones culturales de la población mexicana, en toda su diversidad; así como a la promoción de las competencias que hagan socialmente significativo su uso.

\section{BIBLIOGRAFÍA}

BARTOLOMÉ, M. A. (1997), Gente de costumbre y gente de razón: las identidades étnicas en México. México: Siglo Veintiuno.

BECERRA, M. y MASTRINI, G. (2004), "La Sociedad de la Información en la Argentina: una mirada desde la economía política”, Estudos de Sociologia, 17: 97-113.

BELL, D. (1976), El advenimiento de la sociedad post-industrial. Madrid: Alianza Editorial.

BERGONZELLI, P. y COLOMBO, S. (2006), "La Sociedad de la Información y el Conocimiento: implicancias para América Latina”. Contribuciones a la Economía, http://www.eumed.net/ce/2006/ pbsc.htm.

BOURDIEU, P. (2006), “Génesis y estructura del campo religioso”. Relaciones, 108, XXVII: 29-83.

BURKI, S. J. y PERRY, G. (1998), Más allá del Consenso de Washington: la hora de la reforma institucional. Washington: Banco Mundial.

CABELLO, R. y MOYANO, R. (2006), “TIC y Educación. Competencias Tecnológicas y capacitación para la apropiación de las tecnologías”. Razón y Palabra, núm. 46. http:// www.razonypalabra.org.mx/anteriores/n49/bienal/Mesa\%2013/PonenciaRoxanaCabello2.pdf.

[CEPAL] Comisión Económica para América Latina (2014), Los Pueblos Indígenas en América Latina. Santiago, Chile: Naciones Unidas.

CROVI, D. (2002), "Sociedad de la información y el conocimiento. Entre el optimismo y la desesperanza”, Revista Mexicana de Ciencias Políticas y Sociales, 185: 13-33. 
DAWKINS, R. (1976), The Selfish Gene. Nueva York: Oxford University Press.

DE LA TORRE, R. y GUTIERRÉZ, C. (2007) (coords.), Atlas de la diversidad religiosa en México. México: CIESAS, COLJAL, COLEF, COLMICH, UQROO, SEGOB, CONACYT.

DÍAZ-COUDER, E. (1998), "Diversidad cultural y educación en Iberoamérica”. América, 1,15.http:// www.ineaformate.conevyt.org.mx/index.php? option=com_docman\&task=doc_download\&gid=448\&Itemid=52.

FERNÁNDEZ CHRISTLIB, F. (2013), El transfondo emocional de la comunicación interpersonal (y el difícil tránsito hacia la comunicación intersubjetiva).

GEERTZ, C. (1996), Los usos de la diversidad. Barcelona: Paidós.

GUZMÁN, A. y GÓMEZ, H. (2010), “Brechas tecnológicas y procesos de convergencia entre países emergentes industrializados en la industria farmacéutica”, en Guillén, A. (coord.). Una década de estudios sobre economía social: 39-80. México: UAM Iztapalapa / Juan Pablos Editor.

GÓMEZ PALACIO, C. (1998), Comunicación y Educación en la Era Digital. Retos y Oportunidades. México: Diana.

HERNÁNDEZ, A. M. (1983), "Los grupos étnicos y los sistemas tradicionales de poder en México”. Nueva Antropología. Revista de Ciencias Sociales, (20), pp. 5-30.

INEGI (2010a). “Lenguas indígenas en México y hablantes (de 5 años y más) al 2010”. http:// cuentame.inegi.org.mx/hipertexto/todas_lenguas.htm.

INEGI (201b), Panorama sociodemográfico de México. México: INEGI.

INTERNET WORLD STATS (2013), “Mexico and Central America”. http:// www.internetworldstats.com/central.htm.

JIMÉNEZ, A. y GUTIÉRREZ, M. G. (2010), “Software libre como alternativa para desarrollar sistemas informáticos basados en la lengua indígena de la población. El caso de OpenBiblio distribución náhuatl". Revista Apertura, núm. especial marzo de 2010. http:// www.udgvirtual.udg.mx/apertura/index.php/apertura3/article/view/132/134.

KRIPPENDORFF, K. (1990), Metodología de análisis de contenido. Teoría y práctica. Barcelona: Paidós. LEY GENERAL DE DERECHOS LINGÜÍSTICOS PARA LOS PUEBLOS INDÍGENAS (2003), Diario Oficial de la Federación, 13 de marzo de 2003. http://www.dof.gob.mx/nota_detalle.php? codigo $=698625 \&$ fecha $=13 / 03 / 2003$.

MATTELART, A. (2002), Historia de la Sociedad de la Información. Barcelona: Paidós.

MARX, L. (2001), “Information technology in historical perspective”, en En Schön, D., Sanyal, B. y Mitchell, W. (Eds.) High technology and low-income communities, Cambridge, Massachussets: MIT Press: $131-148$.

MIÈGE, B. (2002), "La société de l'information toujours aussi inconcevable”. Revue européenne des sciences sociales, XL, núm. 123: 41-54.

MILLÁN, M. A. y RIZO, M. (eds.), La comunicación humana en tiempos de lo digital, México: UAM-C / Juan Pablos Editor / AMIC.

MUMFORD, L. (1967), The myth of machine. Nueva York: Harcourt Brace Jovanovich.

NEGROPONTE, N. (1995), Ser Digital. México: Editorial Océano.

OCAMPO, J. A. (2005), "Más allá del Consenso de Washington: una agenda de desarrollo para América Latina". CEPAL Estudios y perspectivas. Núm. 26: 5-30. 
PÉREZ SALAZAR, G. (2004), Análisis crítico del Sistema Nacional eMéxico: la estrategia web del gobierno federal para la reducción de la brecha digital (tesis de maestría). México: Universidad Nacional Autónoma de México.

PÉREZ SALAZAR, G. (2007), “Evaluación del Programa de Acceso a Servicios Digitales en Bibliotecas Públicas: una perspectiva desde la brecha digital”. Global Media Journal [en línea]. Primavera 2007, Vol. 4, No. 7. Disponible en: http://gmje.mty.itesm.mx/perez_salazar.html.

PÉREZ SALAZAR, G. (2009), "Hacia una tecnología socialmente significativa”. En Santos, M. J. y De Gortari, R. (coords.) Computadoras e Internet en la biblioteca pública mexicana, México: UNAM - IIS / Pearson: 1-26.

PÉREZ SALAZAR, G. y CARABAZA, J. (2011), “El Sistema Nacional e-México a diez años de distancia: un nuevo discurso con bajos niveles de interacción”. Versión. Estudios de Comunicación y Política, núm. 27, UAM-Xochimilco.

RAPOSO, M.; FUENTES, E. y GONZÁLEZ, M. (2006), “Desarrollo de competencias tecnológicas en la formación inicial de maestros". Revista Latinoamericana de Tecnología Educativa, 5 (2): 525537. http://www.unex.es/didactica/RELATEC/ sumario_5_2.htm.

RITZER, G. (2007), Blackwell encyclopedia of sociology. Malden, MA: Blackwell Publishing.

SANTOS, M. J.; DE GORTARI, R.; ANGULO, Y.; RUEDA, E. y PÉREZ SALAZAR, G. (2006), Acceso Tecnológico: Una reinterpretación de la biblioteca pública mexicana. México: Conaculta.

STALLMAN, R. (2002), “Free software definition”, en Gay, J. (Ed.) Free Software, Free Society: Selected essays of Richard M. Stallman. Boston: Free Software Foundation, Inc.

SZASZ, I. (1998), “Sexualidad y género: algunas experiencias de investigación en México”. Debate feminista, 9 (18): 77-104.

TODOROV, T. (2007), Nosotros y los otros. México: Siglo Veintiuno.

TOFFLER, A. (1993), El shock del futuro. Barcelona: Plaza y Janés.

[UIT] Unión Internacional de Telecomunicaciones (2005), Cumbre Mundial sobre la Sociedad de la Información. Documentos Finales. Ginebra: UIT.

VILAS, C. M. (2000). “Más allá del ‘Consenso de Washington’? Un enfoque desde la política de algunas propuestas del Banco Mundial sobre reforma institucional". Revista del CLAD Reforma y Democracia. No. 18

WARSCHAUER, M. (2003), "Informatización y desarrollo humano". Scientific American Latinoamerica. 2 (15): 18-23.

\section{NOTAS}

[5月. El autor desea agradecer a la M.E. Sonia O. Martínez Ayala por la corrección de estilo hecha al texto.

1. Como hemos planteado aquí mismo, reconocemos plenamente la existencia de otros posibles indicadores de dicha diversidad, sin embargo, la lengua constituye un articulador observable de otro tipo de condiciones que refieren el complejo cultural en que se sitúa el sujeto, que se manifi esta de manera inevitable en toda interacción social.

2. País identificado por la CEPAL (2014), como el cuarto en América Latina en diversidad de grupos autóctonos. 
3. Vilas (2000) señala las siguientes acciones, que son además la base para el neoliberalismo: 1) disciplina fiscal; 2) priorización del gasto público en áreas de alto retorno económico; 3) reforma tributaria; 4) tasas positivas de interés fi jadas por el mercado; 5) tipos de cambio competitivos y liberalización financiera; 6) políticas comerciales liberales; 7) apertura a la inversión extranjera; 8) privatizaciones; 9) desregulación amplia; 10) protección a la propiedad privada.

4. El Sistema Nacional e-México es mencionado por primera vez, durante el discurso de toma de posesión de Vicente Fox como Presidente de México, en diciembre de 2000. Sin embargo, durante todo 2001 el proyecto careció de una asignación presupuestal específica y no fue sino hasta 2002 que realmente arrancó como iniciativa gubernamental.

5. Periodo que comprende las administraciones de Vicente Fox Quesada (2000-2006) y Felipe Calderón Hinojosa (2006-2012) al frente del poder ejecutivo federal en México; ambos pertenecientes al Partido Acción Nacional.

6. El planteamiento hablaba del establecimiento de un Network Access Point y un backbone de alta velocidad con recursos públicos. Al momento de escribir este trabajo, se hablaba de la posibilidad de incorporar la red de fibra óptica instalada por la empresa paraestatal Comisión Federal de Electricidad (CFE), como base para dicha infraestructura de conectividad, que actuara como alternativa a las troncales privadas establecidas por empresas del sector de las telecomunicaciones, entre las que destaca Teléfonos de México (propiedad de Grupo CARSO).

7. Según Stallman (2002) el software libre se define a partir de cuatro libertades básicas: 1) libertad para emplear el software con cualquier propósito, 2) libertad para conocer la forma en que fue codificado, 3) libertad para hacer cualquier modificación y 4) libertad para distribuirlo.

8. Término empleado durante la administración federal del Lic. Vicente Fox Quesada (2000-2006), para referirse a personas mayores de 60 años.

9. El enlace llevaba a una página de la Presidencia de la República, que albergaba la Ley Indígena, aprobada el 28 de abril de 2001 por el Congreso mexicano.

10. Hasta el momento en que fue elaborado este trabajo, dicha iniciativa se encontraba disponible en una subpágina de la Presidencia de la República: http://www.presidencia.gob.mx/edn/. Esta iniciativa sustituye al Sistema Nacional e-México que, como ya ha sido señalado, estuvo presente en el periodo 2000-2012.

11. Este portal cuenta con un traductor automatizado al inglés, francés, alemán, italiano y ruso; pero no a alguna lengua indígena.

12. Disponible en: http://nah.wikipedia.org/wiki/Cal\%C4\%ABxatl.

13. El sitio web de esta organización puede ser consultado en: http://www.mozilla-mexico.org/.

14. Paquetes de extensión (plugins) descargables en: http://www.mozilla-mexico.org/firefox-enlenguas-indigenas/.

15. Según cifras de Internet World Stats (2013), a septiembre de 2012, México tenía un estimado de 38463860 usuarios de Facebook, que representan alrededor del 75\% del total de usuarios de Internet en este país.

16. Agradecemos al Dr. Ignacio Pérez Barragán, profesor de náhuatl en la Facultad de Ciencias Políticas y Sociales de la UNAM, por su orientación en este sentido.

17. https://www.facebook.com/groups/224863081048919/.

18. https://www.facebook.com/groups/161406687356957/.

19. https://www.facebook.com/groups/126674084136044/.

20. Con base en Dawkins (1976), podemos decir que un meme es una unidad cultural que es replicada al interior de un grupo determinado, de manera que forma parte de su acervo. Puede ser tanto una frase, como una acción o una forma simbólica, entre las que se incluyen las imágenes que ilustran el caso del que se habla.

21. La búsqueda se hizo a partir del motor de Facebook, y fueron considerados sólo grupos abiertos y perfiles personales dedicados a la promoción y/o uso de estas lenguas. Los grupos cerrados quedaron fuera de la muestra por limitaciones metodológicas. Esta no pretende ser una 
lista exhaustiva, por lo que es probable que existan muchos otros similares, en estas y otras lenguas autóctonas habladas en territorio mexicano.

22. https://www.facebook.com/LenguaMaya.

23. https://www.facebook.com/mayayucateca.

24. https://www.facebook.com/lenguajemayakiche.

25. https://www.facebook.com/AprendiendoTzeltal.

26. https://www.facebook.com/groups/sanmarcostulija/.

27. https://www.facebook.com/pages/Zapoteco-para-Principiantes/1375385796017271.

28. https://www.facebook.com/ZapotecoPetapa.

29. https://www.facebook.com/yoloelva.kualtakat.5.

30. https://www.facebook.com/groups/106008256155487/.

31. Conviene recordar que en esta entidad se encuentra Cancún, un destacado destino turístico reconocido a nivel internacional, y que forma parte de la llamada Riviera Maya.

\section{RESÚMENES}

Las políticas públicas desarrolladas en México con la intención de integrar su población a la llamada Sociedad de la Información, han presentado una serie de limitaciones muy relevantes. Desde una perspectiva centrada en la diversidad lingüística, en este trabajo se hace una revisión de las estrategias que han sido implementadas en el periodo 2000-2012 por el gobierno federal mexicano, para la incorporación de las Tecnologías de la Información y la Comunicación como elemento central en el desarrollo social. Se plantea que estas acciones han tenido un punto de partida tecnodeterminista y que no han atendido de manera suficiente las condiciones estructurales prevalecientes. Las omisiones del gobierno mexicano en este sentido, coinciden con una notable participación en línea de diversos sectores de la sociedad civil en la generación de contenidos, aplicaciones y el uso activo de lenguas autóctonas presentes en territorio mexicano.

As políticas públicas desenvolvidas no México com a intenção de integrar a sua população na chamada Sociedade da Informação têm apresentado uma série de limitações muito relevantes. A partir de uma perspetiva centrada na diversidade linguística, este trabalho apresenta uma revisão das estratégias que foram implementadas no período de 2000-2012 pelo governo federal mexicano para a incorporação das tecnologias da informação e comunicação como elemento central no desenvolvimento social. Argumenta-se que estas ações têm tido um ponto de partida tecnodeterminista e que não atenderam de maneira suficiente às condições estruturais prevalentes. As omissões do governo mexicano neste sentido coincidem com uma notável participação em linha de diversos setores da sociedade civil na geração de conteúdos, aplicações e uso de línguas autóctones presentes no território mexicano.

Public policies towards Mexico's inclusion in the Information Society, have shown significant limitations. This work addresses Federal Government's strategies, from a linguistic diversity point of view, during 2000-2012. We recognize in those efforts, a clear techno determinism bias, and the lack of efficient actions oriented to the reduction of the Digital Divide. Along with these findings, is evident that some civil society sectors are engaging themselves in the online development of contents, software, as well as a very active usage of native languages present in Mexico's territory, in social networks sites. 
ÍNDICE

Keywords: Information Society, diversity, public policy, civil society

Palavras-chave: Sociedade de Informação, diversidade, políticas públicas, sociedade civil

Palabras claves: Sociedad de la Información, diversidad, políticas públicas, sociedad civil

\section{AUTOR}

\section{GABRIEL PÉREZ SALAZAR}

Universidad Autónoma de Coahuila. Enderezo de correspondencia: Sin Nombre de Colonia, Monclova,COAH, México

gabrielperezsalazar@gmail.com 